\title{
Study On The Barycenter Position Of Measurement And Data Processing For Dura-Axle Vehicle
}

\author{
Bo Wang ${ }^{1, a^{*}}$, Yu-ming Nie ${ }^{1, b}$, Zezhong Yang ${ }^{1, c}$, Chengqiang Zong ${ }^{1, d}$, Lanxin \\ Geng $^{1, e}$
}

${ }^{1}$ Key Laboratory of Operation Safety Technology on Transport Vehicles, Ministry of Transport, PRC, Research Institute of Highway Ministry of Transport, Beijing, Courtyard 8\#, Xitucheng Road, Haidian

District,100088,China

ab.wang@rioh.cn, ${ }^{\mathrm{d}} \mathrm{cq} . z o n g @$ rioh.cn, ${ }^{\mathrm{e} x l . g e n g @ \text { rioh.cn }}$

Keywords: Dura-axle vehicle, barycenter position, least squares method

\begin{abstract}
Base on measuring principle of barycenter, the authors analyzed influencing factors of barycenter and measured barycenter of one Dura-axle vehicle according to GB/T 12538-2003. The result showed that the height value of barycenter is different when lifting vehicle for smaller angle, so when measuring the height of barycenter to avoid lifting smaller angle. The authors processed actual barycenter data using the method of least squares to fitting. The height value of barycenter is uniqueness and accuracy by calculating the data after fitting.
\end{abstract}

\section{Introduction}

Vehicle's barycenter is very important to the driving direction's stability, especially when braking during bend of road. To the design and layout of the vehicle, the accurate measurement of the barycenter of vehicle is of great importance. For passenger vehicle, the barycenter has great influence on handling stability, ride comfort and driving safety. In recent years, frequent rollover accidents of passenger vehicle are of a great relationship with high barycenter. The position of barycenter affects maximum stable roll angle and rollover test energy [1-3]. Therefore, more and more attention is paid to the accurate measurement of the barycenter by related department.

\section{Three Parameters Of Barycenter Measurement Principle}

Longitudinal And Lateral Position Of Barycenter Measurement. The Fig.1 showed that the barycenter parameters consist of $x$ (barycenter position and front axle distance), $y$ (barycenter position and longitudinal symmetrical plane of vehicle distance) and $\mathrm{z}$ (the height of barycenter position). The $m_{1}, m_{2}$, $m_{3}, m_{4}$ were FL, FR, RL, RR wheel load. The following equation can be got by torque balance ${ }^{[4]}$.

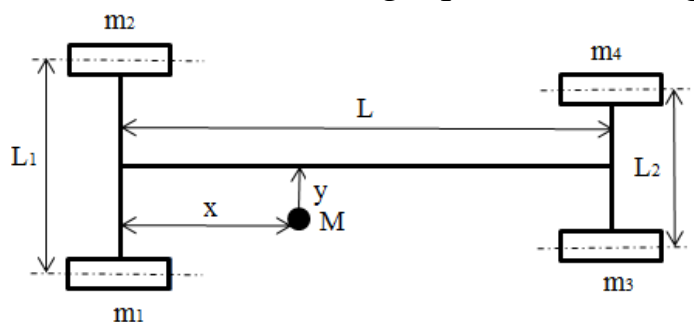

Fig. 1 The diagram of longitudinal and lateral position of barycenter

$$
\begin{gathered}
\left(m_{1}+m_{2}\right) \times x=\left(m_{3}+m_{4}\right) \times(L-x) \\
m_{1} \times\left(\frac{L_{1}}{2}-y\right)+m_{3} \times\left(\frac{L_{2}}{2}-y\right)=m_{2} \times\left(\frac{L_{1}}{2}+y\right)+m_{4}\left(\frac{L_{2}}{2}+y\right)
\end{gathered}
$$

The upper equation can be got by simplified following equation.

$$
x=\frac{\left(m_{3}+m_{4}\right)}{M} \times L
$$




$$
y=\frac{\left(m_{1}-m_{2}\right) \times L_{1}+\left(m_{3}-m_{4}\right) \times L_{2}}{2 \times M}
$$

Where, $\mathrm{M}$ is vehicle mass, $\mathrm{L}$ is the distance between front and rear axles, L1 and L2 are the front wheel-base and rear wheel-base.

Barycenter Position Measurement. The height of barycenter can be measured according to GB/T 1538-2003. Where, $\mathrm{r}$ is tire static radius, $\mathrm{z}_{1}$ is the distance between barycenter and axis connection. So $\mathrm{z}$ is defined by the following equation:

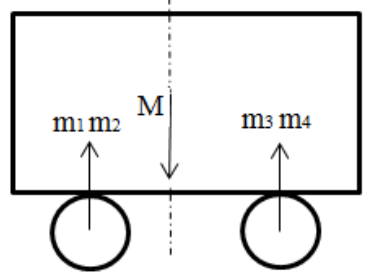

Fig. 2 Diagram in horizontal position

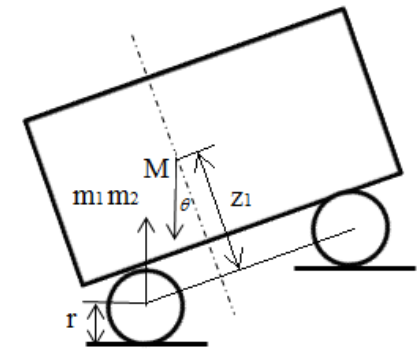

Fig. 3 Diagram when lifting $\theta$

$$
z=z_{1}+r
$$

The Fig. 3 showed that the following equation can be got by torque balance when the lifting angle of vehicle is $\theta$ :

$$
M \times \cos \theta \times(L-x)+M \times \sin \theta \times z_{1}=\left(m_{1}+m_{2}\right) \times \cos \theta \times L
$$

The upper equation can be simplified following equation:

then

$$
z_{1}=\frac{\left(m_{f}{ }^{\prime}-m_{f}\right) \times \mathrm{L}}{M \times \tan \theta}
$$

$$
Z=\frac{\left(m_{f}{ }^{\prime}-m_{f}\right) \times L}{M \times \tan \theta}+r
$$

Where, $m_{f}{ }^{\prime}$ was front wheel load when lifting rear axle and $m_{f}$ was front wheel load when vehicle was horizontal.

\section{Analyzing Influence Factors Of Barycenter Position}

In the three parameters of barycenter, $\mathrm{x}$ and $\mathrm{y}$ can be calculated easily and accurately. But the height of barycenter can be influenced by many factors.

The equation set (8) showed that the height of barycenter was in relation to tire static radius, lifting angle, wheel load variation, wheel base and gross mass of vehicle ${ }^{[5]}$.

The Influence Of The Measuring Equipment. (1) The equation set (8) showed that the height of barycenter and the wheel load difference between before and after lifting was a linear correlation of wheel base (L) times. Therefore, the demand of wheel load equipment is very high. The measurement accuracy of absolute wheel load value is $\pm 0.2 \%$ and the measurement accuracy of wheel load difference is $\pm 2.5 \%$ according to standard. The measurement should be done in flat ground. (2) The axle which is lifted by lifting equipment must be in parallel with the ground to ensure the wheel only by the vertical force. (3) Length and angle measuring instrument should be meet the accuracy requirements, when the length measuring range is less than or equal to $2000 \mathrm{~mm}$, the absolute accuracy of length measuring instrument is $\pm 1 \mathrm{~mm}$; when the length measuring range is more than $2000 \mathrm{~mm}$, the relative accuracy of instrument is $\pm 0.05 \%$; the accuracy of angle measuring instrument is $\pm 1 \%^{[6]}$.

The Influence Of Vehicle. (1) Elastic element of vehicle: the wheel load changed when lifting vehicle, so the tire, suspension of the vehicle and so on deformed, the deformation of elastic element caused measurement error. (2) Liquid of vehicle: such as brake oil, fuel, lubricating oil and so on, moved when measuring. It’s assignable error source. 


\section{Data Processing}

Analyzing The Method Of Data Processing. The vehicle was supposed to be rigid body and the barycenter position cannot change as wheel load changing. Therefore, the height of barycenter is constant when measuring according to GB/T 12538-2003. If the equation of $m_{f}{ }^{\prime}-m_{f} \neq \mathrm{k} \tan \theta$ (k is constant) is workable, the height of barycenter will be decrease as lifting angle increase. Therefore, the height of barycenter cannot change only when the equation of $m_{f}{ }^{\prime}-m_{f}=\mathrm{k} \tan \theta$ is workable. The following equation can be got:

$$
m_{f}{ }^{\prime}=\mathrm{k} \tan \theta+m_{f}
$$

The wheel load and tangent lifting angle is a linear correlation when lifting according to equation set (9), Wheel load can be corrected through least square fitting method when measuring.

Verification Test. Test vehicle is a small bus and following table showed the parameter of the test vehicle.

Table 1 The parameter of the test vehicle

\begin{tabular}{|c|c|c|c|c|c|}
\hline $\begin{array}{l}\text { Gross } \\
\text { mass/ M }\end{array}$ & $\begin{array}{l}\text { tire } \\
\text { static } \\
\text { radius /r }\end{array}$ & $\begin{array}{l}\text { Wheel } \\
\text { base/L }\end{array}$ & $\begin{array}{l}\text { Front/rear } \\
\mathrm{L}_{1} / \mathrm{L}_{2}\end{array}$ & $\begin{array}{l}\text { FL/FR } \\
\text { wheel } \quad 1 \\
\mathrm{~m}_{1} / \mathrm{m}_{2}\end{array}$ & $\begin{array}{l}\mathrm{RL} / \mathrm{RR} \\
\text { wheel load } \\
\mathrm{m}_{3} / \mathrm{m}_{4}\end{array}$ \\
\hline $3368 \mathrm{~kg}$ & $350 \mathrm{~mm}$ & $3920 \mathrm{~mm}$ & $1615 \mathrm{~mm} / 1500 \mathrm{~mm}$ & $827 \mathrm{~kg} / 793 \mathrm{~kg}$ & $946 \mathrm{~kg} / 802 \mathrm{~kg}$ \\
\hline
\end{tabular}

The result that $x=2034 \mathrm{~mm}, y=40 \mathrm{~mm}$ can be calculated through the equation set (3) and set (4).

The front wheel load can be got when lifting rear axle. Table2 showed the front wheel load value.

Table 2 The front wheel load value when meauring

\begin{tabular}{cccc}
\hline Lifting angle $\theta /^{\circ}$ & $\begin{array}{c}\text { Tangent angle } \\
\tan \theta\end{array}$ & $\begin{array}{c}\text { Actual front } \\
\text { wheel load/kg }\end{array}$ & $\begin{array}{c}\text { The height of } \\
\text { barycenter }\end{array}$ \\
\hline 2 & 0.035 & 1634 & 817 \\
7 & 0.123 & 1678 & 900 \\
8 & 0.140 & 1684 & 880 \\
9 & 0.158 & 1690 & 865 \\
10 & 0.176 & 1697 & 858 \\
17.3 & 0.311 & 1755 & 854 \\
\hline
\end{tabular}

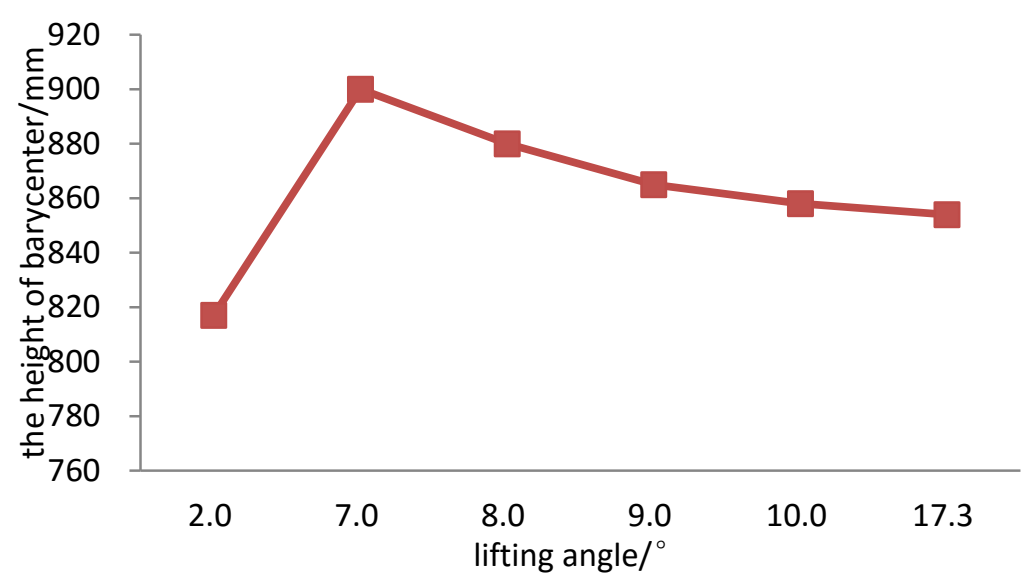

Fig.4 The graph of relativity between the barycenter height and lifting angle

The Table 2 and Fig.4 showed that the height of barycenter that was calculated by measuring date was great error. The maximum error reached $83 \mathrm{~mm}$. That's because the variable quantity of front wheel load was small and load data measured by wheel load meter had great error, therefore, the height of barycenter is not accuracy. It's better to avoid to lift small angle when measuring.

The wheel load and tangent lifting angle is a linear correlation, so it's a straight line in graph and the line was through pint $\left(0, m_{f}\right)$. The authors processed actual barycenter data using the method of least 
squares to fitting. The paper set fitting equation for $y=k x+b$, so the $\mathrm{b}=1620$ will be got and also $\mathrm{k}=438.6$ will be got. Therefore, the fitting equation is $y=438.6 x+1620$. The following table showed the wheel load before and after fitting.

Table3 Front wheel load before and after fitting

\begin{tabular}{cccc}
\hline Lifting angle $\theta /^{\circ}$ & $\begin{array}{c}\text { Tangent angle } \\
\tan \theta\end{array}$ & $\begin{array}{l}\text { Actual wheel } \\
\text { load/kg }\end{array}$ & $\begin{array}{l}\text { Wheel } \\
\text { after fitting/kg }\end{array}$ \\
\hline 8 & 0.140 & 1684 & 1681.61 \\
9 & 0.158 & 1690 & 1689.43 \\
10 & 0.176 & 1697 & 1697.30 \\
17.3 & 0.311 & 1755 & 1756.54 \\
\hline
\end{tabular}

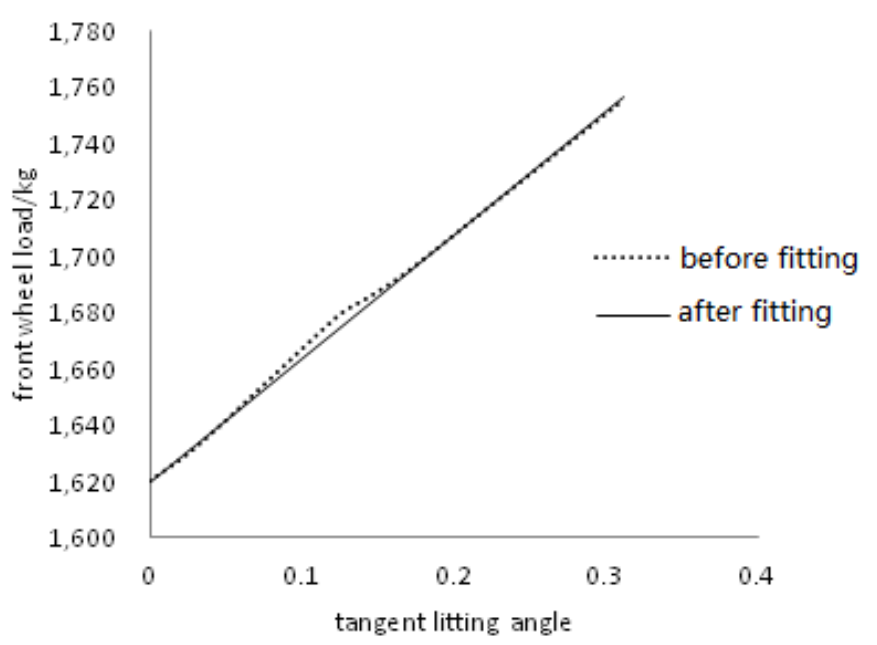

Fig. 5 Wheel load before and after fitting

The height of barycenter was $860.5 \mathrm{~mm}$ that can be calculated by equation set (8) through the front wheel load after fitting. The unique height of barycenter can be got by the method of least squares to fitting and it was more accurate. Likewise, the height of barycenter can be got by lifting front axle.

\section{Conclusions}

By the height of barycenter calculation formula, it can be learned that the biggest factor that affects barycenter height is the accuracy of measurement of axle load.

It is very convenient to measure the barycenter height by the usage of lifting axle method of GB/T 12538-2003. However, it should be avoided that the lifting angle is too small. When the lifting angle is small, the smaller the axle load changes, the bigger the deviation of measurement of the wheel load meter is. Therefore it is recommended that the lifting height should be not less than 10 degree.

It is feasible to use least square method for fitting axle load values which are actually measured. The value of barycenter height which is calculated by fitting is unique and its accuracy is ensured.

\section{References}

[1] Peng Chen, Shenjie Hao, Zhenzhong Li. Data Processing Method of Gravity Height for Dual-axle Vehicle. Auto Engineer.2014(5) 47-50.

[2]Houlin Li, Hua Tian. Study on Load Table Mass Transfer In Measuring Vehical Gravity Center Height By Roll Testing Method. Drive System Technique.1999:19-25.

[3] Dongming Xie, Bin Qiu. Study on Measuring Method and Data Processing Analysis of Centre of Gravity for Two-axle Vehicle [J].Automobile Technology, 2009(4):45-49

[4] Xiaofeng Bao, Debang Ding. Center of gravity of motor-vehicle, Measuring and test 
technology.1997(11):21-25.

[5] Jianru Li, Zhaochen Wu. Application of Mass Reaction Method in Measuring the Height of Vehicle Center of Gravity. Auto Engineer.2010(11):54-55.

[6] Heshen Yin. Gravity Reaction Method, Height of the Center of Gravity Measurement. Automobile Technology. 1993:16-18. 